\title{
Status and Prospects of High-Power Heterostructure Barrier Varactor Frequency Multipliers
}

\author{
By Jan Stake, Aleksandra Malko, Tomas Bryllert, and Josip Vukusic
}

ABSTRACT | There is a high demand for compact, roomtemperature sources operating at millimeter-wave and terahertz (THz) frequencies for space instruments and terrestrial applications. This part of the electromagnetic spectrum is by far the least explored because of the difficulty of generating energy at these frequencies. Continuous-wave oscillators based on either electronics or photonics are limited in output power for fundamental reasons. Varistor and varactor frequency multipliers have shown outstanding performance in terms of output power, but further technical development will be essential to solve the lack of efficient and compact terahertz sources. In this paper, we present the status of heterostructure barrier varactor (HBV) diode frequency multipliers. The performance and prospects for THz applications in which HBV diode technology can offer advantages over conventional solutions are discussed. For instance, such a device can be easily scaled by increasing the number of barriers to produce and handle higher power. The inherent symmetry confines the power generation to odd harmonics, thereby simplifying the design of high-order frequency multipliers. For example, highpower triplers $(\times 3)$, quintuplers $(\times 5)$, nonlinear transmission lines (NLTLs) and grid multipliers utilizing HBV diodes are presented. Overall, HBV technology is a natural stepping stone from high-power microwave amplifiers to higher frequencies and can both simplify and improve the performance of terahertz sources.

KEYWORDS | Frequency conversion; heterostructure barrier varactors (HBVs); millimeter-wave diodes; III-V semiconductors; semiconductor heterojunctions; submillimeter-wave diodes; terahertz sources; varactors

\footnotetext{
Manuscript received April 12, 2016; revised September 22, 2016; accepted November 29, 2016

The authors are with the Department of Microtechnology and Nanoscience, Chalmers University of Technology, SE-41296 Göteborg, Sweden. (e-mail: jan.stake@chalmers.se).
}

\section{INTRODUCTION}

Efficient and compact terahertz (THz) sources are crucial for emerging terahertz applications in communication, remote sensing, and spectroscopy. At present, the relatively low output power of $\mathrm{THz}$ sources due to the difficulty of generating energy at these frequencies limit the dynamic range and acquisition speed of $\mathrm{THz}$ instruments. In particular, between the microwave and far-infrared domains of the electromagnetic spectrum (approximately 0.3-3 THz) [1], there is a lack of compact, efficient continuous-wave sources [2] that can generate sufficient signal power $(\mathrm{mW})$ to drive room-temperature $\mathrm{THz}$ heterodyne mixers [3]. Hence, to further expand the use of $\mathrm{THz}$ waves outside the traditional niche of space science, there is a strong need for solutions to close the "THz gap," that is, to realize high-performance, compact, and reasonably inexpensive transmitter components.

Fundamental photonic sources, such as the solid-state quantum cascade laser, require cryogenic temperatures when operating at these relatively long wavelengths, suffer from low tunability, and require frequency stabilization for narrow-line applications. Frequency multipliers, which are based on harmonic generation in nonlinear devices, provide excellent spectral purity, narrow line widths, and high power levels compared with fundamental oscillators (e.g., Gunn diodes) [4] or optical mixing in photodiodes (e.g., uni-traveling-carrier photodiodes) [5]. Moreover, varactor multipliers can exhibit a relatively high conversion efficiency [6], whereas frequency multipliers of the varistor type are less efficient but are suitable for broadband signal generation [7], [8]. Recent progress in transistor and monolithic microwave integrated circuit (MMIC) technology has pushed the frontier for active multiplier chains into the submillimeter wavelength range [9]. However, the output power degrades rapidly with increasing frequency, and low levels of parasitic 
loss and coupling are critical for operation beyond $1 \mathrm{THz}$. The Schottky diode (SD), a two-terminal device, exhibits low parasitics because of its simple structure and is therefore currently the workhorse for both mixer and varactor applications at THz frequencies [10]. A chain of multipliers is typically necessary to reach $\mathrm{THz}$ frequencies, thus necessitating the development of high-order multipliers to minimize the number of cascaded components.

A high multiplication factor can be achieved with devices that exhibit symmetric capacitance-voltage (C-V) characteristics and thus generate only odd harmonics of the pump frequency signal. These characteristics simplify the design of high-order frequency multipliers (e.g., $\times 3, \times 5$ ). For example, two SD varactors integrated and connected in anti-series exhibit symmetry [11]. Device concepts in which the symmetry is a part of the semiconductor material stack and device layout have also been proposed. Progress on the growth of compound semiconductor materials (III-Vs) during the 1970s and 1980s has allowed several junctions to be epitaxially stacked and has enabled devices to be tailored for certain applications. The heterostructure barrier varactor (HBV) diode, which consists of a stack of semiconductors, where heterojunctions between adjacent layers creates the electrical function, is one such device.

The HBV diode was developed based on research on harmonic generation in resonant tunneling diodes (RTDs) [12]. An RTD device consists of a double barrier structure, where the discrete electron energy levels within the quantum well give rise to nonlinear I-V characteristics with a negative differential resistance region. The negative resistance is vital for oscillator applications but is not beneficial in varactor applications, such as harmonic generation. In 1989, Kollberg [13] and Rydberg at the Chalmers University of Technology proposed a quantum barrier varactor (QBV) device [14], where a wide bandgap semiconductor (barrier) prevents conduction current through the diode and low bandgap semiconductors with a moderate n-doping concentration (modulation layers) on each side of the barrier give rise to nonlinear symmetric $\mathrm{C}-\mathrm{V}$ characteristics. The first QBV frequency tripler was presented by Rydberg et al. in 1990 [15], demonstrating the potential of this device for odd harmonic generation up to $300 \mathrm{GHz}$. Both QBVs and single-barrier varactors (SBVs), referring to the same device concept, appeared in the literature during the early 1990s. These terms, particularly SBV, were replaced with the term HBV when multiple-barrier structures ( $N$-barriers) were introduced. The possibility of adding several varactor diode junctions in series, which are stacked during epitaxial growth, is a key advantage that permits the scaling of the power handling capability $\left(\mathrm{N}^{2}\right)$ without adding loss and physical size due to additional contacts compared to a conventional multidiode topology.

During the first decade of HBV research, the primary focus was on the growth and design of the material stack, fabrication, and modeling of the device. The first devices
Table 1 Standard HBV Layer Structure

\begin{tabular}{|c|c|c|c|c|}
\hline No & Layer & Material & Thickness & Doping Level \\
\hline 10 & Contact & InAs & $\sim 10 \mathrm{~nm}$ & $\sim 10^{19} \mathrm{~cm}^{-3}$ \\
\hline 9 & Contact & $\mathrm{In}_{0,53} \mathrm{GaAs}$ & $\sim 400 \mathrm{~nm}$ & $\sim 10^{19} \mathrm{~cm}^{-3}$ \\
\hline 8 & Modulation & $\mathrm{In}_{0,53} \mathrm{GaAs}$ & $300 \mathrm{~nm}$ & $1 \times 10^{17} \mathrm{~cm}^{-3}$ \\
\hline 7 & Spacer & $\mathrm{In}_{0,53} \mathrm{GaAs}$ & $5 \mathrm{~nm}$ & Undoped \\
\hline 6 & Barrier & $\mathrm{In}_{0,52} \mathrm{AlAs}$ & $5 \mathrm{~nm}$ & Undoped \\
\hline 5 & Barrier & AlAs & $3 \mathrm{~nm}$ & Undoped \\
\hline 4 & Barrier & $\mathrm{In}_{0,52} \mathrm{AlAs}$ & $5 \mathrm{~nm}$ & Undoped \\
\hline 3 & Spacer & $\mathrm{In}_{0,53} \mathrm{GaAs}$ & $5 \mathrm{~nm}$ & Undoped \\
\hline 2 & Modulation & $\mathrm{In}_{0,53} \mathrm{GaAs}$ & $300 \mathrm{~nm}$ & $1 \times 10^{17} \mathrm{~cm}^{-3}$ \\
\hline 1 & Contact/buffer & $\mathrm{In}_{0,53} \mathrm{GaAs}$ & $\sim 500 \mathrm{~nm}$ & $\sim 10^{19} \mathrm{~cm}^{-3}$ \\
\hline 0 & Substrate & InP & & Semi-insulating \\
\hline
\end{tabular}

were made in the AlGaAs/GaAs material system, and whisker contacts were used to access a single HBV mesa. The first planar HBV diode, with airbridges, was demonstrated by the team at University of Virginia in 1997 [16]. A problem at that time was the relatively high leakage current due to the poor electron barrier in the AlGaAs/GaAs material system [17], which degraded the conversion efficiency [18] when used in frequency multipliers (hybrid varactor-varistor mode). Several methods to improve the barrier height were proposed; however, the main advancement came with lattice-matched InGaAs/InAlAs HBVs grown on InP. Several HBV triplers with excellent conversion efficiency were achieved because of the superior electron blocking characteristics and lower series resistance due to the high mobility and low bandgap of InGaAs. In particular, the group at IEMN in Lille demonstrated the first HBV tripler with a conversion efficiency above $10 \%$ at $250 \mathrm{GHz}$ [19]. Since 2000, the main achievements have involved integrated HBV circuits, with highpower triplers [20], quintuplers [21], and novel multiplier topologies being developed based on nonlinear transmission lines [22] and large HBV grids [23]. In this review paper, we present a historical overview, the basic concepts, and the current status of HBV multipliers for THz applications.

\section{DEVICE PRINCIPLES AND PARAMETERS}

\section{A. Basic Principles and Device Design}

The HBV is a unipolar and symmetric device. The active device unit is composed of an undoped high-bandgap semiconductor barrier that is sandwiched between moderately doped low-bandgap semiconductors (modulation layers) (see Table I). This unit block, i.e., layer sequences 2-7, can be repeated $N$ times to create an $N$-barrier HBV. The remaining part of the material stack is required to provide low access resistance and good ohmic contacts. The heterojunctions forming the barrier are designed to prevent electron transport through the diode, resulting in a device operating in varactor mode over a wide range of large-signal (pumping) and operating conditions [24]. Hence, the barrier should be undoped, high, and sufficiently thick to minimize thermionic 


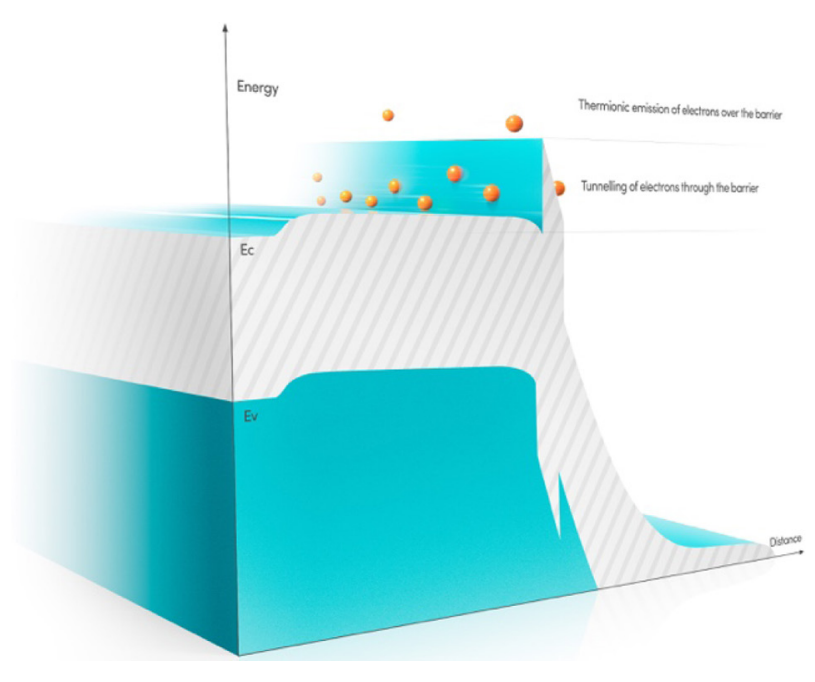

Fig. 1. Energy band diagram showing the mechanisms of carrier transport through a typical HBV barrier structure.

emissions and carrier tunneling [25], as shown in Fig. 1. The effective barrier height can be improved with a thin pseudomorphic (3 nm) AlAs layer in the center of the barrier [26], suppressing the leakage current even further [27].

With an applied electric field, the capacitance is modulated due to the extension of a depleted charge region. This results in a nonlinear $\mathrm{C}-\mathrm{V}$ curve [28], as shown in Fig. 2. The $\mathrm{C}-\mathrm{V}$ curve is an even function of the applied voltage due to the symmetry of the device structure. In varactor applications, the differential elastance, $S=\partial V / \partial Q=1 / C$, is often used to analyze the performance under large signal excitation.

For frequency multiplication with nonlinear capacitors, the conversion efficiency is mainly limited by a parasitic series resistance $R_{s}$ and the capacitance modulation during a pump cycle. An important figure of merit is the dynamic cutoff frequency of the diode

$$
f_{c}=\frac{S_{\max }-S_{\min }}{2 \pi R_{s}}
$$

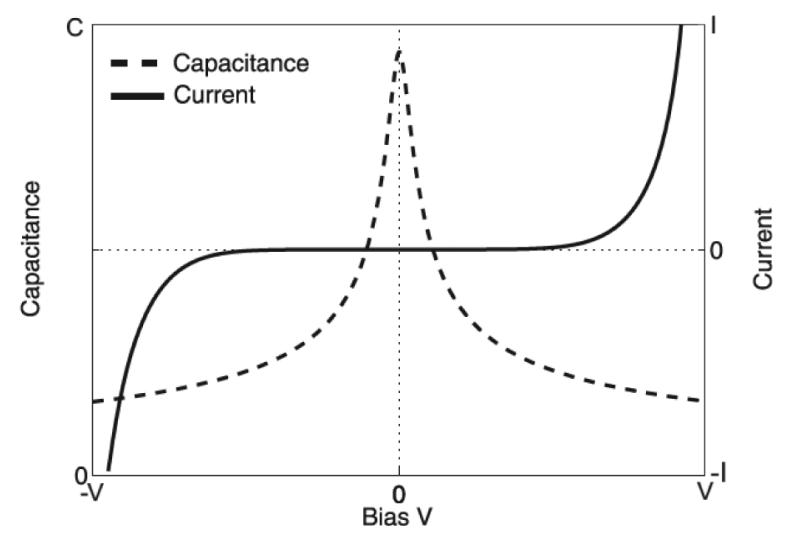

Fig. 2. Typical I-V and C-V characteristics of the HBV diode. where $S_{\max }$ and $S_{\min }$ are the maximum and minimum differential elastances, respectively, during a pump cycle [29]. For high efficiency, any varactor diode must exhibit low series resistance $R_{s}$ and accommodate a large elastance swing, i.e., a high $S_{\max } / S_{\min }$ ratio. Hence, the design of the epilayer structure of the HBV diode typically begins by determining the doping concentration and layer thicknesses via optimization of the dynamic cutoff frequency [30]. To achieve a high $S_{\max } / S_{\min }$ ratio, carriers one each side of the barrier can be brought closer to the barrier by doping of the spacer region or via quantum wells adjacent to the barrier [31]. The minimum capacitance $C_{\min }$ is typically obtained for the punch-through condition (the modulation layers are completely depleted of carriers at one point during the pump cycle), for high-frequency current saturation (further depletion of the modulation layers is prohibited due to the finite saturation velocity of electrons) [32], or when the maximum voltage $V_{\max }$ is reached ( $V_{\max }$ is limited by the device reliability and the onset of current conduction).

\section{B. Device Models}

Using the quasi-static equivalent circuit model shown in Fig. 3, the voltage across the nonlinear capacitor for homogeneously doped HBVs can be expressed as a function of its charge as

$$
\begin{aligned}
v(Q, T)= & \frac{N}{A}\left(\frac{b Q}{\varepsilon_{b}}+\frac{2 s Q}{\varepsilon_{d}}+\operatorname{sign}(Q)\right. \\
& \left.\left(\frac{Q^{2}}{2 \varepsilon_{d} q N_{d} A}+\frac{4 k_{B} T}{q} A\left(1-e^{-\frac{|Q|}{2 L_{D} q N_{d} A}}\right)\right)\right)
\end{aligned}
$$

where $T$ is the device temperature, $q$ is the elementary charge, $k_{B}$ is Boltzmann's constant, $L_{D}$ is the Debye length, and $Q$ is the charge stored in the HBV [33]. For a simple analysis of symmetric varactors [34], the voltage versus charge can be described by a Taylor series $\left(v=\alpha Q+\beta Q^{3}+\ldots\right)$ with only odd terms [35]. The associated series resistance $R_{s}$

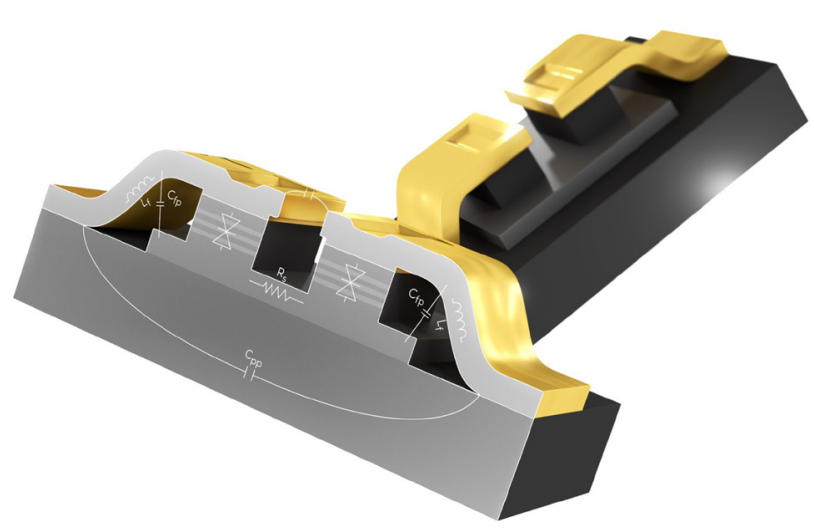

Fig. 3. Planar HBV diode layout and equivalent circuit elements. 


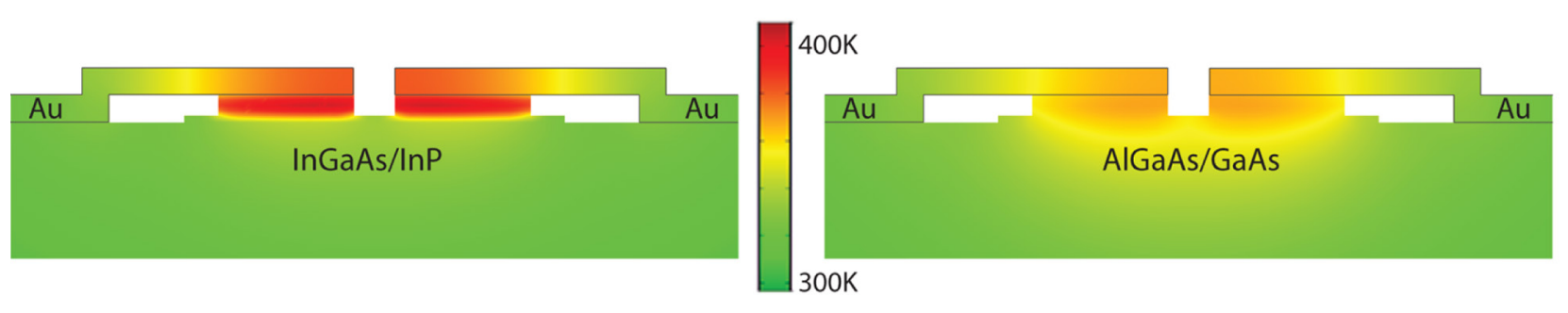

Fig. 4. Simulated (FEM) temperature profile for typical planar HBV MMICs with two 200- $\mu \mathrm{m}^{2}$ mesas: (left) InGaAs-based and (right) AlGaAs-based HBV.

summarizes the resistive losses that characterize various layers and connections forming the device. The main contributions to the series resistance are the ohmic contact resistance, the spreading resistance, and the resistance in the modulation layers (intrinsic part). Given the voltagecharge relationship described in (2), the series resistance can be expressed as [30]

$$
\begin{aligned}
R_{s}(Q, T, f)= & R_{s 0}(T, f)-\frac{\rho_{d} N}{A} \\
& \times\left(\frac{|Q|}{q N_{d} A}+2 L_{D}\left(e^{-\frac{|Q|}{2 L_{D} q N_{d} A}-1}\right)\right)
\end{aligned}
$$

where $R_{s 0}(T, f)$ is the zero-bias series resistance and $\rho_{d}$ is the resistivity of the modulation layer

$$
\rho_{d}=\frac{1}{q N_{d} \mu_{e}\left(T, N_{d}\right)} .
$$

The extrinsic spreading resistance in the contact regions of the device is dependent on the resistivity, geometry, and frequency through the skin [36] and proximity effects [37], [38]. One disadvantage of the HBV diode is the requirement for two low ohmic contacts, whereas the SD requires only one ohmic contact. However, for a multibarrier HBV, the extrinsic part of the series resistance (spreading, contacts, etc.) will be shared among all active junctions, and the dynamic cutoff frequency $f_{c}$ will increase with the number of epitaxial stacked barriers. By contrast, in the SD case, the dynamic cutoff frequency will not improve with several anodes in a series configuration. From this perspective, it is clear that HBV technology is competitive when several junctions are required, e.g., for power applications.

Under normal varactor operation, the conduction current typically plays a minor role as long as the total current $i(t)$ is dominated by the displacement current $\partial Q / \partial t$. It is difficult to model the conduction current with a physical analytical expression because of the mixture of thermionic emissions and tunneling through the barrier. Typically, an empirical model based on the $\sinh (x)$ function can describe the I-V characteristics [39]. For high power and drive levels, the effect of self-heating [18] is quite severe because the mobility degrades and conduction current increases at elevated junction temperatures. A complete electrothermal model (Chalmers HBV Model) was presented by Ingvarson et al. [40]. Self-consistent solutions of semiclassical semiconductor transport equations [41], [42] or extensions of the equivalent circuit model are often used for a more refined analysis and optimization.

\section{Power Handling and Thermal Management}

HBV devices for high power generate heat due to ohmic losses. Because excessive heat is detrimental to the performance and longevity of electronic devices, a successful high-power design must include efficient thermal management. Thermal management involves considerations of the device and circuit layout and the choice of the substrate material. The thermal aspect is particularly constraining for InGaAs because of its low heat conductivity $(\kappa=4.6 \mathrm{~W} / \mathrm{m})$, which is a factor of ten lower than in GaAs. This circumstance will create a high thermal gradient within the active device region for InGaAs-based HBVs. This high thermal gradient effectively limits the maximum number of barriers that can be stacked for increased power handling in an HBV [43]. Assuming an ideal case, where the active region is surrounded with perfect heat sinks, the maximum number of barriers that can be stacked for a certain temperature raise is proportional to $\sqrt{\kappa}$ [43]. Consequently, the material system determines the maximum output power from an HBV multiplier because the conversion efficiency $\left(f_{c}\right.$, varactor versus varistor mode) and the power handling capability $\left(N^{2} \propto \kappa\right)$ are all related to the material properties, such as carrier mobility, thermal conductivity, and conduction band offset (barrier). The difference between InGaAs and AlGaAs HBV MMICs from a thermal handling perspective for an equivalent power dissipation of $200 \mathrm{~mW}$ is illustrated in Fig. 4. The benefit of a high-thermal-conductivity substrate (e.g., diamond, silicon, AlN) plays a minor role in InGaAs HBVs due to the material's poor heat conductivity [44].

\section{Design and Optimization}

Optimization of the HBV device design for maximum output power involves the consideration of several 
limitations: the maximum voltage over the barriers, maximum current density, maximum junction temperature, and practical impedance range for the embedding circuit. If the maximum voltage is surpassed, then the ensuing leakage current will reduce performance and might even cause irreversible device damage. This is also true if the device is operated above the maximum temperature. However, in this case, there might be long-term repercussions that affect performance, i.e., reliability. For high current densities, current saturation [32] also hinders the performance. In addition, there is the practical consideration of embedding impedance, which is difficult to realize for low HBV device impedances. When performing the simulations to optimize the device parameters, mainly the device area and number of barriers, the aforementioned limitations together with the available pump power and frequency are used as the boundary conditions [45]. The optimization process necessitates that the electrical and thermal aspects are self-consistently included [40].

\section{Fabrication of HBV Diodes and Circuits}

The main issue involved in HBV device realization is the ability to combine low- and high-bandgap semiconductor materials, as detailed in Table 1. Due to the progress in material growth techniques during the last decades, particularly the molecular beam epitaxy (MBE) technique, semiconductor alloys of different compositions can be stacked with atomic monolayer precision. These growth methods allow for abrupt heterojunctions with low defect density, thereby ensuring low leakage current and high-voltage handling, which is essential for efficient HBV multiplication.
As noted above, HBVs were initially realized in the $\mathrm{AlGaAs} / \mathrm{GaAs}$ material system, which is lattice matched over the entire $\mathrm{Al}_{\mathrm{x}} \mathrm{Ga}_{1-\mathrm{x}} \mathrm{As}$ range. In addition, the $\mathrm{AlGaN} / \mathrm{GaN}$ material system, with its superior thermal conductivity and large heterostructure band offsets, has been explored [46], [47], in addition to the low-bandgap system AlSb/InAs [48]. However, improved performance was achieved using the more mature InGaAs/InAlAs semiconductor compound alloy because of its higher mobility and moderate bandgap offset. Unfortunately, this material system, with InP as the host substrate, is more challenging to grow because it is not lattice matched. This issue of lattice matching has limited the number of grown $(\leq 3)$ barriers and overall thickness that can be achieved with this material.

Table 1 lists a standard layer sequence that is optimized for a high breakdown voltage per barrier [27]. The pseudomorphic AlAs layer is only $3 \mathrm{~nm}$ thick, and although the highbandgap AlAs layer increases the barrier height, its lattice mismatch limits the total thickness of the grown structure. The barrier layers are left undoped to increase the effective barrier height and thus voltage handling. An undoped spacer layer separates the barrier from the modulation layer. III-V MBE materials are typically grown at temperatures exceeding $500{ }^{\circ} \mathrm{C}$, thereby avoiding diffusion of dopant species into the barrier layer that would compromise the barrier height.

After the material growth is finalized, standard III-V fabrication methods, such as lithography, wet and dry etching, and metal deposition, are used to fabricate the diodes and connected circuits. As an example, a step-bystep account of the device fabrication for the double-mesa HBV is shown in Fig. 5. First, the contacts are defined by
1

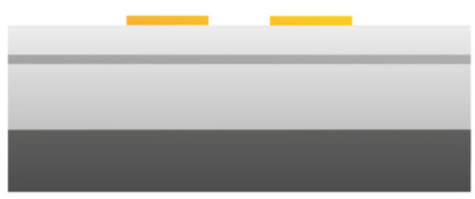

4

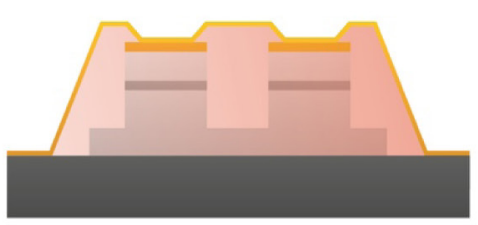

2

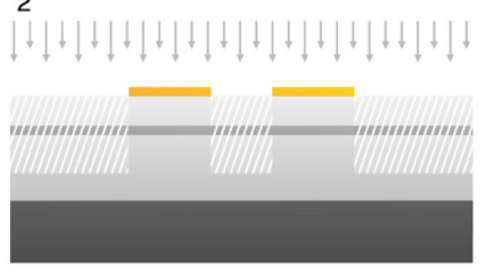

5

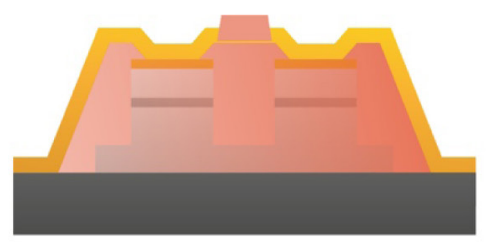

3

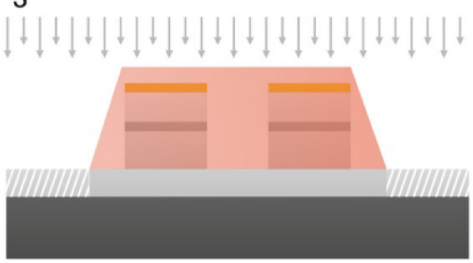

6

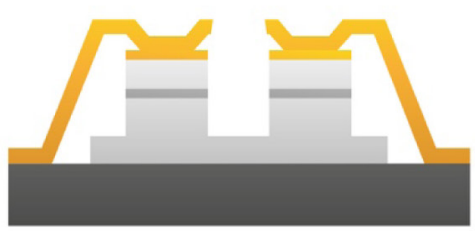




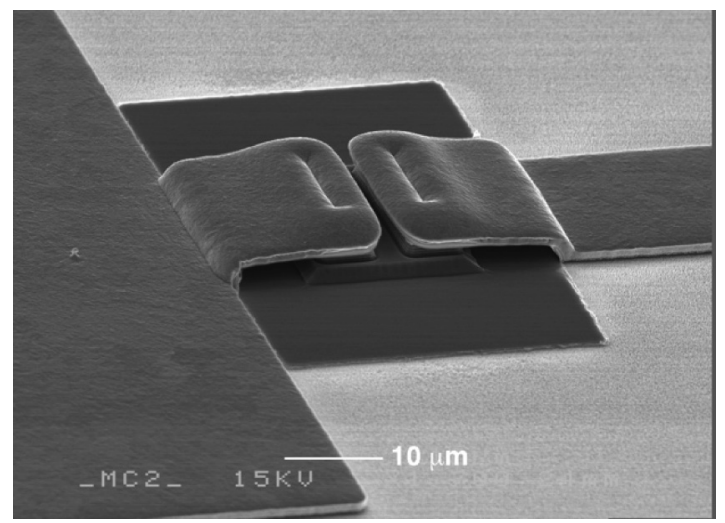

Fig. 6. SEM photograph of an air-bridged, 2-mesa $\mathrm{HBV}$ device with a total of six barriers.

photolithography and metal contact evaporation [Fig. 5(1)]. Typically, NiGeAu is used for the ohmic contact; however, TiPtAu was also investigated for HBV devices. In addition to the contact metallization, a layer of titanium is evaporated. This layer is used as a hard mask for the dry etching of the diode mesas (down to layer 1 in Table 1) in a $\mathrm{CH}_{4}$ atmosphere [Fig. 5(2)].

After the second lithographical patterning step, the InGaAs buffer layer is removed in an $\mathrm{H}_{2} \mathrm{O}_{2}: \mathrm{H}_{3} \mathrm{PO}_{4}: \mathrm{H}_{2} \mathrm{O}$ etchant solution [Fig. 5(3)]; hence, an electrical isolation between contact pads is obtained. A photoresist layer is then patterned and covered with a gold seed layer [Fig. 5(4)] for the electroplating [Fig. 5(5)] of the connecting pads. This sacrificial layer of photoresist and thin gold is then removed to complete the device fabrication, as shown in Fig. 5(6). The scanning electron microscopy (SEM) image in Fig. 6 shows a 2-mesa HBV diode with electroplated gold connections.

In the case of monolithic integration, when the wafer substrate is used as a waveguide, the microstrip circuit is gold electroplated in the same step [Fig. 5(5)] as the HBV device. A monolithically integrated circuit is shown in Fig. 7.

The completed devices are thinned to the designed substrate thickness, and then, diced and separated. For more accurate control of the final circuit thickness, a method based on epitaxial transfer onto a silicon-on-insulator (SOI) substrate with a predefined thickness has been developed [21]. In this method, instead of growing the HBV structure on silicon, the epitaxial material is transferred onto the host substrate (Fig. 8) using one of several possible wafer bonding methods, e.g., spin-on glass [49], [50] or plasma activation [51].

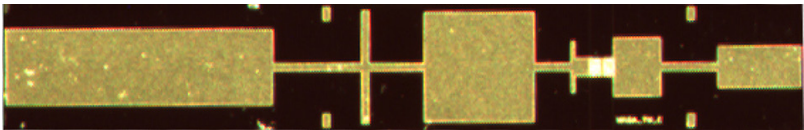

Fig. 7. Photograph of a monolithically integrated HBV multiplier circuit.

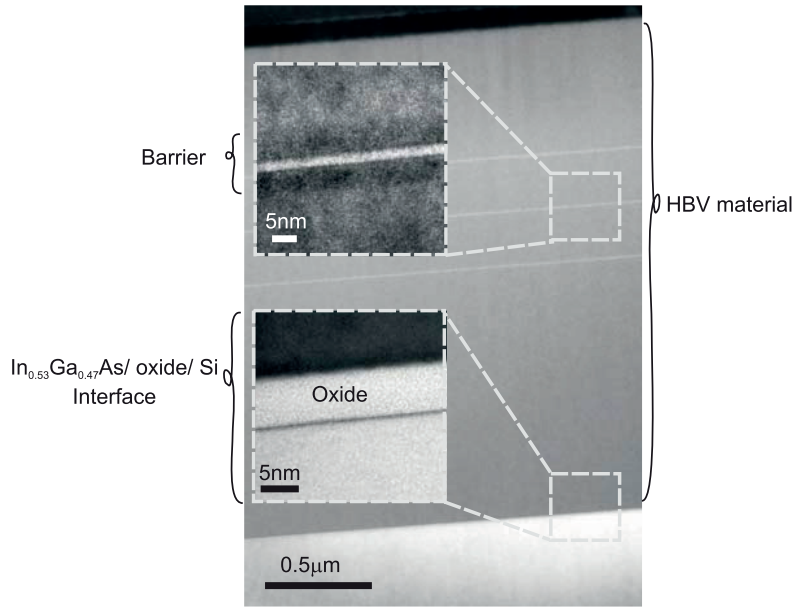

Fig. 8. Transmission electron microscopy image of the HBV material structure epitaxially transferred onto a silicon substrate [52].

\section{CHARACTERIZATION}

\section{A. I-V and C-V Characterization}

The nonlinear $\mathrm{C}-\mathrm{V}$ characteristics can be measured using a standard low-frequency impedance meter (e.g., impedance bridge), as long as the small-signal susceptance $\omega C$ is not negligible compared to the conductance $G=\partial \mathrm{I} / \partial \mathrm{V}$. Extraction of the I-V characteristics from direct current (dc) measurements is a straightforward process. To resolve the low current levels and features in the low-bias regime, pA-nA accuracy or better is required. Within the safe bias range of HBVs, the junction or barrier resistance is typically extremely high compared to the series resistance, making it difficult to extract the series resistance from I-V measurements, as often applied to SDs biased into the forward region. Hence, broadband S-parameter measurements [53] are required to extract the complete equivalent circuit, including the important series resistance. Nevertheless, for diodes with high Q-values, the uncertainty in $R_{s}$ from parameter extraction and highfrequency S-parameter measurements is high [54].

\section{B. Reliability Aspects and Failure Conditions}

For both ground and space applications, it is necessary to estimate the lifetime and possible failure mechanisms. To date, few investigations have been conducted on HBV reliability. To the authors' knowledge, only limited device studies initiated by the European Space Agency have been conducted. For any high-power RF device, it is important to assess and mitigate various failure mechanisms due to elevated temperatures. Passivation of the mesa side walls is also crucial to withstand any environmental stress that may affect the device characteristics. From a varactor frequency multiplier application perspective, there are several important parameters that influence the performance: the symmetry of the $\mathrm{C}-\mathrm{V}$ characteristic; the maximum and minimum capacitances $C_{\max }$ and 


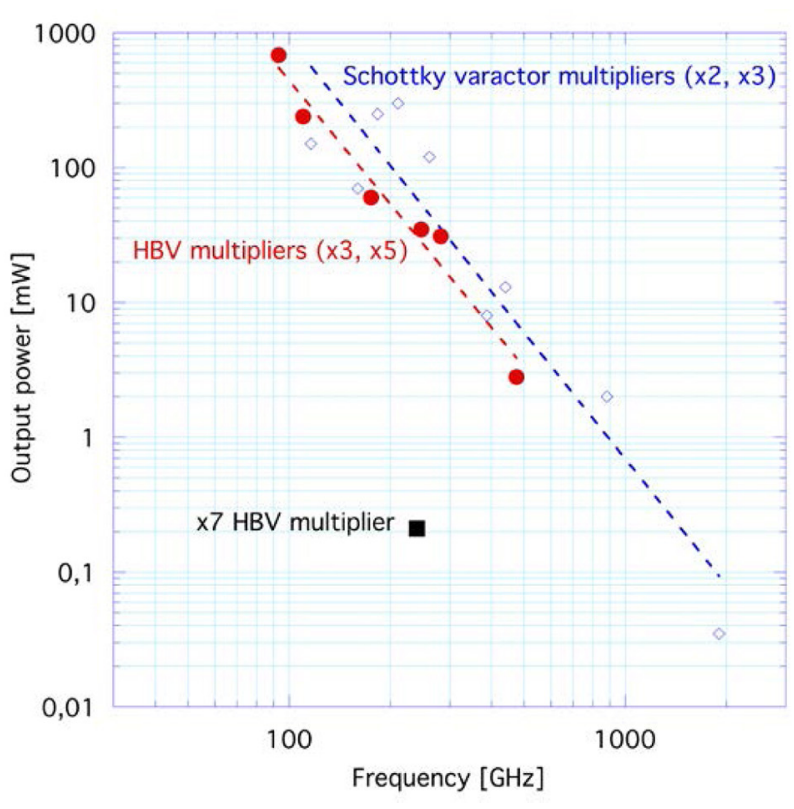

Fig. 9. Current status of the output power for HBV and Schottky varactor diode frequency multipliers [20], [21], [23], [55], [66].

$C_{\min }$, respectively; the conduction current $(\mathrm{I}-\mathrm{V})$; the maximum or breakdown/maximum voltage $V_{\max }$; and the series resistance $R_{s}$. True lifetime assessment and qualification of HBV multipliers requires thorough RF and mechanical stress tests of actual modules; hence, such tests are designed with a certain application in mind. For general process development, a simplified reliability assessment based on electrical measurements and optical inspections at the device level can provide insight into failure mechanisms. For varactors, monitoring over time the following electrical parameters with appropriate failure conditions (e.g., a 10\%-20\% change) can be used to investigate the device reliability:

- differential capacitance at zero bias $C_{\max }$;

- maximum voltage defined as the voltage at a certain current density, e.g., $J_{\max }=25 \mathrm{nA} / \mu \mathrm{m}^{2}$;

- minimum differential conductance $(d I / d V)$ at zero bias $G_{\min }$; this parameter is highly sensitive to any change in the barrier region or leakage current within the device structure;

- series resistance $R_{s}$, which is a highly relevant parameter that is difficult to extract without $S$-parameter measurements and parameter extraction methods.

\section{HBV Circuits}

\section{A. State-of-the-Art Triplers and Quintuplers}

Fig. 9 summarizes the current state-of-the-art diode frequency multipliers based on SD and HBV devices. These frequency multipliers include both hybrid and monolithic integrated circuits and different levels of power combining.
Comparing SD-based with HBV-based frequency multipliers, there are two main aspects that make devices of the second type more attractive from a circuit design perspective:

- because of the symmetrical $\mathrm{C}-\mathrm{V}$ characteristic, the HBV generates only the odd harmonics of the incoming signal $\left(f_{p}\right)$, thereby facilitating a high multiplication factor;

- the frequency multipliers operate symmetrically at $0 \mathrm{~V}$ and do not require external biasing or dc ground.

Hence, the circuit design is greatly simplified and can be made to be extremely compact.

The inherent suppression of the even harmonics in the output spectrum indicates that a frequency tripler will not require an idler circuit. Circuit ground connections (e.g., via holes, solder points, and beam leads) and $\mathrm{dc}$ lines are often difficult to achieve at high frequencies, and the fact that these connections are not required simplifies both the circuit design and the circuit processing and assembly. Without this galvanic connection, the device is also protected from electrostatic discharge (ESD), which is beneficial from a reliability perspective. However, removing the possibility of biasing and monitoring the device makes tuning during operation impossible. Triplers have been demonstrated at frequencies of up to $450 \mathrm{GHz}$ [67]. The majority of circuits are designed for high-power applications requiring limited bandwidth [55] ; however, circuits with a relative bandwidth of 15\%-20\% have also been demonstrated [68]. A number of circuits operating in the 100-120-GHz frequency band have demonstrated the high-power properties of HBV circuits with an output power of $240 \mathrm{~mW}$ [67] from a single HBV device. Similarly, an output power of $30 \mathrm{~mW}$ has been achieved at $282 \mathrm{GHz}$ [20], also using a single device. This performance is possible because multiple HBV heterostructures can be stacked epitaxially in the semiconductor material, thereby increasing the maximum power of operation.

A typical HBV frequency tripler is fabricated as a waveguide-coupled microstrip circuit (Fig. 10). The circuit consists of input/output waveguide probes and impedance matching structures for the fundamental frequency and third harmonic. Because there is no isolation between the input side and output side in a diode circuit, a stop filter for the third harmonic is required at the input side and a stop filter is required for the fundamental frequency side at the output side. The stop filter for the fundamental frequency is typically achieved through the low-frequency cutoff of the output waveguide, whereas the filter for the third harmonic is typically achieved using circuit elements, such as a stepped impedance low-pass filter or a $\lambda / 4$ stub filter, as shown in Fig. 10.

Because of their power handling capability and compactness, HBV triplers are attractive as general-purpose millime- 
a)

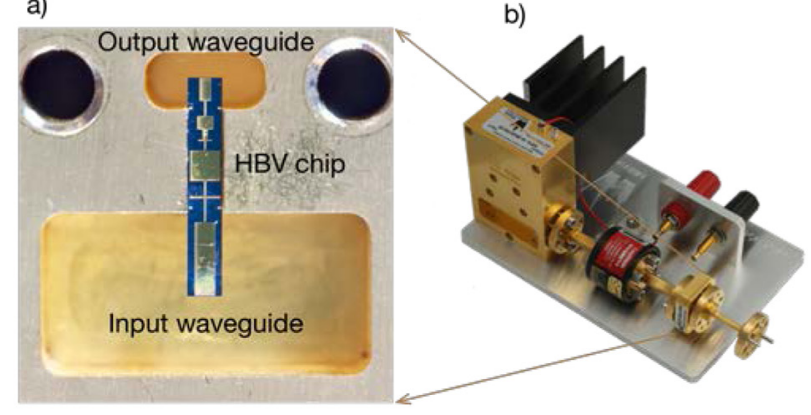

Fig. 10. (a) Integrated HBV frequency multiplier assembled in a waveguide block. (b) A 300-GHz high-power source (HBV $\times 3 /$ GaN power amplifier).

ter-wave sources and, in particular, as part of local oscillator chains for scientific instrumentation [69].

The first HBV quintupler $(\times 5)$ was demonstrated by Räisänen et al. in 1995 [70], showing the advantage of high-multiplication factors using symmetric varactors. The absence of even harmonics simplifies the design; however, the intermediate harmonic $3 x f_{\mathrm{p}}$ must be terminated properly. For the highest possible quintupler conversion efficiency, this idler circuit [71] is ideally a reactive termination that maximizes the third harmonic current. Compared with an SD-based solution $(\times 2$ or $\times 3)$, the HBV quintupler $(\times 5)$ will have a large impact if it can replace two doublers in cascade. It is difficult to achieve broadband operation in a high-order multiplier due to the requirement of simultaneous impedance matching at various harmonics (input, idlers, and output), spanning over several octaves. Hence, it is logical to use high-order multipliers for high-power, narrowband applications. In particular, the quintupler solution is a suitable stepping stone to reach higher frequencies (THz), i.e., as a first multiplier stage followed by a second-stage Schottky doubler/tripler. State-of-the-art HBV quintuplers deliver more than $60 \mathrm{~mW}$ at $175 \mathrm{GHz}$ [62] and a few milliwatts at approximately $470 \mathrm{GHz}$ [21]. From a circuit perspective, higher harmonic multiplication factors $(>\times 5)$ are increasingly difficult to implement, despite a $240-\mathrm{GHz}$ septupler ( $\times 7)$ having been successfully demonstrated by the team at the University of Virginia (Charlottesville, VA, USA) [56]. In Table 2, we provide a summary of the performance of the HBV frequency multipliers that have been reported to date.

\section{B. Alternative HBV Circuits}

Quasi-optical circuits are attractive, particularly at extreme frequencies, where the losses in waveguide circuits are higher. A quasi-optical HBV frequency tripler, where two slot antennas loaded with HBVs are located at the focal plane of a dielectric lens, is shown in Fig. 11. In this example, a simultaneous impedance match at the fundamental and third harmonic was achieved [75].

One method of overcoming the fundamental electrical and thermal limitations of single solid-state devices is to coherently combine the emitted radiation, quasioptically, from a large array of $M$ emitting elements [76]. As a first-order approximation, such a 2-D solution generates $M$ times the output power compared with a single device. These types of quasi-optical solutions can also be housed in overmoded waveguides (see Fig. 12) and can be made extremely compact in practice [65]. By spatially power combining $72 \mathrm{HBV}$ diodes in a closed waveguide, Dahlbäck et al. demonstrated the first tripler operating close to submillimeter-wave frequencies [23]. Large multidiode arrays can be analyzed and simplified via a unit-cell approach. However, a fully detailed 3-D model is the ultimate technique for predicting the power

Table 2 HBV Multipliers

\begin{tabular}{|c|c|c|c|c|c|c|}
\hline $\begin{array}{c}\mathbf{f}_{\text {out }} \\
(\mathbf{G H z})\end{array}$ & $\begin{array}{c}\text { Pout } \\
(\mathrm{mW})\end{array}$ & $\eta(\%)$ & $\begin{array}{c}\text { 3-dB bandwidth } \\
(\%)\end{array}$ & $\begin{array}{c}\text { Multiplication } \\
\text { factor }\end{array}$ & Comments & Reference \\
\hline 93 & 684 & 11 & & $\times 3$ & Integrated, 2D-grid & {$[65]$} \\
\hline 100 & 4.5 & 11.4 & 1.1 & $\times 5$ & Hybrid, quartz substrate & [72] \\
\hline 103 & 184 & 23 & 9 & $\times 3$ & Integrated, Si substrate & [52] \\
\hline 107 & 185 & 23 & 15 & $\times 3$ & Integrated, InP substrate & {$[68]$} \\
\hline 110 & 240 & 20 & 6 & $\times 3$ & Hybrid, AlN substrate & [66] \\
\hline 113 & 195 & 15 & 1.5 & $\times 3$ & Hybrid, AlN substrate & {$[55]$} \\
\hline 175 & 60 & 6.3 & 4.5 & $\times 5$ & Hybrid, AlN substrate & {$[62]$} \\
\hline 202 & 20 & 3 & & $\times 5$ & Hybrid, AlN substrate & [66] \\
\hline 240 & 0.21 & 0.25 & - & $\times 7$ & Integrated, InP substrate & {$[56]$} \\
\hline 247 & 35 & 4 & 3 & $\times 3$ & Integrated, 2D-grid & {$[23]$} \\
\hline 282 & 31 & 7 & - & $\times 3$ & MMIC, InP substrate & [20] \\
\hline 288 & 6 & 6 & 15 & $\times 3$ & Integrated, Quartz & [73] \\
\hline 290 & 9.5 & 8 & 8.6 & $\times 3$ & Hybrid, AlN & [74] \\
\hline 450 & 1 & 1.45 & - & $\times 3$ & Hybrid, GaAs & [67] \\
\hline 474 & 2.8 & 0.75 & 4 & $\times 5$ & Integrated, SOI substrate & [21] \\
\hline
\end{tabular}




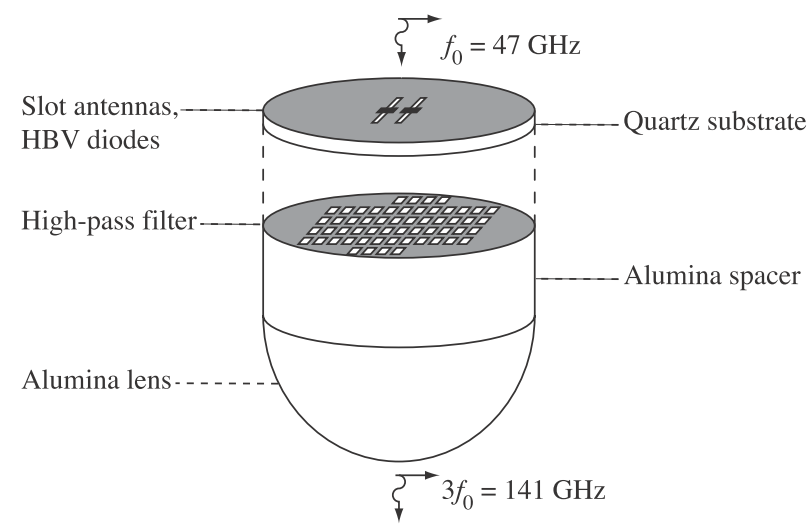

Fig. 11. A 141-GHz integrated quasi-optical slot antenna tripler [75].

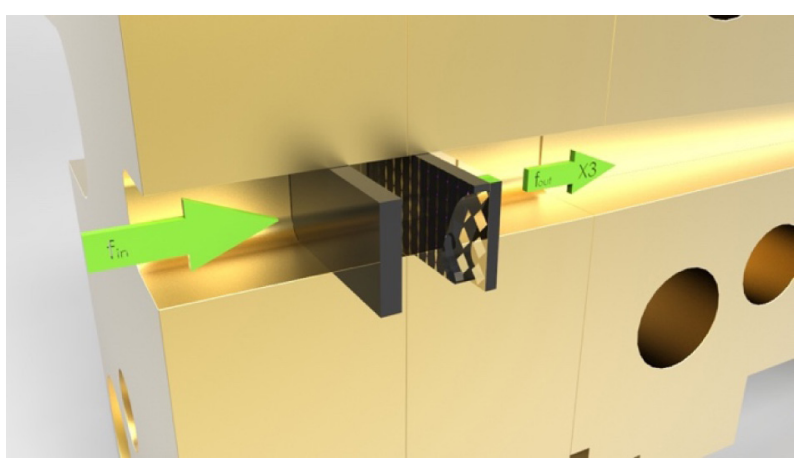

Fig. 12. Illustration of a THz grid HBV multiplier circuit enclosed in a rectangular waveguide cavity.

distribution, spurious modes, and the coupling between the elements [77].

Nonlinear transmission lines (NLTLs) are an interesting approach for realizing high-bandwidth multipliers and pulse generators. The first HBV NLTL tripler was presented by Hollung et al. [22] using 15 discrete diodes, periodically loaded along a finline enclosed in a rectangular waveguide, as shown in Fig. 13. True distributed HBV transmission line circuits have been proposed, but they are difficult to model and real-

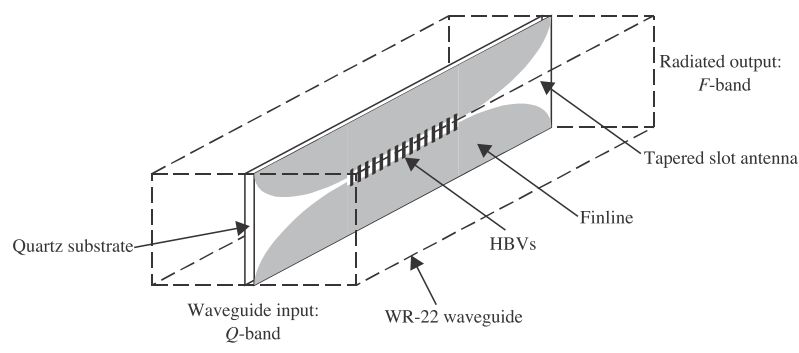

Fig. 13. Schematic of an NLTL HBV tripler in a finline configuration [22]. ize [78]. At present, varactors are mainly used for voltage tuning or as harmonic generators; however, nonlinear reactive devices can also be used as parametric mixers and amplifiers. For example, the symmetry of HBVs can be exploited to realize a subharmonic upconverter, in which the odd sidebands are suppressed [79].

\section{CONCLUSION}

In this paper, we described the history, basic concepts, and advantages and disadvantages of HBV diode technology. We also analyzed and identified applications in which HBV technology can offer advantages over conventional Schottky-based solutions. At frequencies above $150 \mathrm{GHz}$, varactor multipliers are producing state-of-the-art power levels. Although multipliers are primarily based on the more mature Schottky diode technology, HBV-based multipliers have a number of advantages. HBV-based multipliers only generate odd harmonics and can operate without bias, thereby reducing the circuit complexity. The HBV can be easily scaled by increasing the number of barriers to provide higher powers. With the advent of W-band amplifiers producing watts of output power, the HBV tripler $(\times 3)$ to 300 $\mathrm{GHz}$ and the HBV quintupler $(\times 5)$ to $500 \mathrm{GHz}$ appear to be competitive and unique solutions. However, HBV technology must mature in terms of cost, reproducibility, and reliability to meet the requirement for a large fraction of space-borne and ground-based applications.

To summarize, substantial improvements remain to be achieved in the development of HBV technology, including 1) studying new circuit topologies, material composition, and device optimization; and 2) improving integration/manufacturing techniques. The technical potential and cost-effective solutions that HBV technology can provide motivates further research and technical development on high-power and high-order HBV multipliers for use in the $\mathrm{THz}$ frequency range.

\section{Acknowledgments}

J. Stake would like to thank Prof. E. Kollberg for providing an introduction to HBV technology and for highlighting the possibility of exploring HBV devices and circuits as part of his Ph.D. work. The authors would like to thank Dr. T. Närhi and Dr. P. Piironen (ESA/ESTEC, Holland) for their fruitful discussions and long-term support. Finally, they would like to thank and acknowledge their colleagues who have participated in the research on HBVs at Chalmers, including Dr. R. Dahlbäck, G. Hrubó, J. Hanning, Dr. Ø. Olsen, Dr. M. Ingvarson, Dr. A. Emadi, Dr. B. Alderman, Dr. S. Kazemi, M. Sadeghi, C.-M. Kihlman, Dr. S. Hollung, Dr. L. Dillner, Dr. H. Grönqvist, and Dr. S. M. Nilsen. 


\section{REFERENCES}

[1] P. H. Siegel, "Terahertz technology," IEEE Trans. Microw. Theory Techn., vol. 50, no. 3, pp. 910-928, Mar. 2002.

[2] G. Chattopadhyay, "Technology, capabilities, and performance of low power terahertz sources," IEEE Trans. THz Sci. Technol., vol. 1, no. 1, pp. 33-53, Aug. 2011.

[3] H. W. Hubers, "Terahertz heterodyne receivers," IEEE J. Sel. Topics Quantum Electron., vol. 14, no. 2, pp. 378-391, Mar. 2008.

[4] H. Eisele, "State of the art and future of electronic sources at terahertz frequencies," Electron. Lett., vol. 46, no. 26, pp. s8-s11, Dec. 2010.

[5] H. Ito, T. Furuta, F. Nakajima, K. Yoshino, and T. Ishibashi, "Photonic generation of continuous THz wave using uni-travelingcarrier photodiode," J. Lightw. Technol., vol. 23, no. 12, pp. 4016-4021, Dec. 2005.

[6] J. M. Manley and H. E. Rowe, "Some general properties of nonlinear elements-Part I. General energy relations," Proc. IRE, vol. 44, no. 7, pp. 904-913, Jul. 1956.

[7] C. H. Page, "Frequency conversion with positive nonlinear resistors," J. Res. Nat. Bur. Stand., vol. 56, no. 4, pp. 179-182, Apr. 1956.

[8] R. H. Pantell, "General power relationships for positive and negative nonlinear resistive elements," Proc. IRE, vol. 46, no. 12, pp. 1910-1913, Dec. 1958.

[9] A. Zamora et al., "A submillimeter wave InP HEMT multiplier chain," IEEE Microw. Wireless Compon. Lett., vol. 25, no. 9, pp. 591-593, Aug. 2015.

[10] T. W. Crowe, W. L. Bishop, D. W. Porterfield, J. L. Hesler, and R. M. Weikle, "Opening the terahertz window with integrated diode circuits," IEEE J. Solid-State Circuits, vol. 40, no. 10, pp. 2104-2110, Oct. 2005.

[11] M. Krach, J. Freyer, and M. Claassen, "Power generation at millimetre-wave frequencies using GaAs/GaAlAs triplers," Phys. Status Solidi C, vol. 1, no. 8, pp. 2160-2182, Jul. 2004.

[12] T. C. L. G. Sollner, P. E. Tannenwald, D. D. Peck, and W. D. Goodhue, "Quantum well oscillators," Appl. Phys. Lett., vol. 45, no. 12, pp. 1319-1321, 1984.

[13] P. H. Siegel, "Terahertz pioneer: Erik L. Kollberg-'Instrument maker to the stars,"' IEEE Trans. THz Sci. Technol., vol. 4, no. 5, pp. 538-544, Oct. 2014.

[14] E. Kollberg and A. Rydberg, "Quantumbarrier-varactor diodes for high-efficiency millimetre-wave multipliers," Electron. Lett., vol. 25, no. 25, pp. 1696-1698, Dec. 1989.

[15] A. Rydberg, H. Gronqvist, and E. Kollberg, "Millimeter- and submillimeter-wave multipliers using quantum-barrier-varactor (QBV) diodes," IEEE Electron Device Lett., vol. 11, no. 9, pp. 373-375, Sep. 1990.

[16] J. R. Jones, W. L. Bishop, S. H. Jones, and G. B. Tait, "Planar multibarrier 80/240-GHz heterostructure barrier varactor triplers," IEEE Trans. Microw. Theory Techn., vol. 45, no. 4, pp. 512-518, Apr. 1997.

[17] D. Landheer, H. C. Liu, M. Buchanan, and R. Stoner, "Tunneling through AlAs barriers: $\Gamma$-X transfer current," Appl. Phys. Lett., vol. 54, no. 18, pp. 1784-1786, 1989.

[18] J. Stake et al., "Effects of self-heating on planar heterostructure barrier varactor diodes," IEEE Trans. Electron Devices, vol. 45, no. 11, pp. 2298-2303, Nov. 1998.
[19] X. Melique et al., "Record performance of a $250 \mathrm{GHz}$ InP-based heterostructure barrier varactor tripler," Electron. Lett., vol. 35, no. 11, pp. 938-939, 1999.

[20] J. Vukusic, T. Bryllert, Ø. Olsen, J. Hanning, and J. Stake, "Monolithic HBV-based 282GHz tripler with 31-mW output power," IEEE Electron Device Lett., vol. 33, no. 6, pp. 800-802, Jun. 2012.

[21] A. Malko, T. Bryllert, J. Vukusic, and J. Stake, "A $474 \mathrm{GHz}$ HBV frequency quintupler integrated on a $20 \mu \mathrm{m}$ thick silicon substrate," IEEE Trans. THz Sci. Technol., vol. 5, no. 1, pp. 85-91, Jan. 2015.

[22] S. Hollung, J. Stake, L. Dillner, M. Ingvarson, and E. Kollberg, "A distributed heterostructure barrier varactor frequency tripler," IEEE Microw. Guided Wave Lett., vol. 10, no. 1, pp. 24-26, Jan. 2000.

[23] R. Dahlbäck, J. Vukusic, R. M. Weikle, II, and J. Stake, "A tunable $240-290 \mathrm{GHz}$ waveguide enclosed 2-D grid HBV frequency tripler," IEEE Trans. THz Sci. Technol., vol. 6, no. 3, pp. 503-509, Apr. 2016.

[24] A. Uhlir, "The potential of semiconductor diodes in high-frequency communications," Proc. IRE, vol. 46, no. 6, pp. 1099-1115, Jun. 1958.

[25] Y. Fu, J. Stake, L. Dillner, M. Willander, and E. L. Kollberg, "AlGaAs/GaAs and InAlAs/ InGaAs heterostructure barrier varactors," J. Appl. Phys., vol. 82, no. 11, pp. 5568-5572, 1997.

[26] V. K. Reddy and D. P. Neikirk, "High breakdown voltage AlAs/InGaAs quantum barrier varactor diodes," Electron. Lett., vol. 29, no. 5, pp. 464-466, Mar. 1993.

[27] T. A. Emadi, T. Bryllert, M. Sadeghi, J. Vukusic, and J. Stake, "Optimum barrier thickness study for the InGaAs/InAlAs/AlAs heterostructure barrier varactor diodes," Appl. Phys. Lett., vol. 90, no. 1, p. 012108 , Jan. 2007.

[28] Y. Fu, L. Dillner, J. Stake, M. Willander, and E. L. Kollberg, "Capacitance analysis for AlGaAs/GaAs and InAlAs/InGaAs heterostructure barrier varactor diodes," J. Appl. Phys., vol. 83, no. 3, pp. 1457-1462, 1998.

[29] P. Penfield and R. P. Rafuse, Varactor Applications. Cambridge, MA, USA: MIT Press, 1962.

[30] J. Stake, S. H. Jones, L. Dillner, S. Hollung, and E. L. Kollberg, "Heterostructure-barriervaractor design," IEEE Trans. Microw. Theory Techn., vol. 48, no. 4, pp. 677-682, Apr. 2000.

[31] E. Lheurette, X. Melique, P. Mounaix, F. Mollot, O. Vanbesien, and D. Lippens, "Capacitance engineering for InP-based heterostructure barrier varactor," IEEE Electron Device Lett., vol. 19, no. 9, pp. 338-340, Sep. 1998.

[32] E. L. Kolberg, T. J. Tolmunen, M. A. Frerking, and J. R. East, "Current saturation in submillimeter-wave varactors," IEEE Trans. Microw. Theory Techn., vol. 40, no. 5, pp. 831-838, May 1992.

[33] L. Dillner, J. Stake, and E. L. Kollberg, "Modeling of the heterostructure barrier varactor diode," presented at the Int. Semiconductor Device Res. Symp., Charlottesville, VA, USA, 1997, pp. 179-182.

[34] L. Dillner, J. Stake, and E. L. Kollberg, "Analysis of symmetric varactor frequency multipliers," Microw. Opt. Technol. Lett., vol. 15, no. 1, pp. 26-29, Mar. 1997.
[35] K. Krishnamurthi and R. G. Harrison, "Analysis of symmetric-varactor-frequency triplers," in IEEE MTT-S Int. Microw. Symp. Dig., Jun. 1993, pp. 649-652.

[36] H. A. Wheeler, "Formulas for the skin effect," Proc. IRE, vol. 30, no. 9, pp. 412-424, Sep. 1942.

[37] L. E. Dickens, "Spreading resistance as a function of frequency," IEEE Trans. Microw. Theory Techn., vol. 15, no. 2, pp. 101-109, Feb. 1967.

[38] A.-Y. Tang and J. Stake, "Impact of eddy currents and crowding effects on highfrequency losses in planar Schottky diodes," IEEE Trans. Electron Devices, vol. 58, no. 10, pp. 3260-3269, Aug. 2011.

[39] L. Dillner et al., "Frequency multiplier measurements on heterostructure barrier varactors on a copper substrate," IEEE Electron Device Lett., vol. 21, no. 5, pp. 206-208, May 2000.

[40] M. Ingvarson, J. Vukusic, A. Ø. Olsen, T. A. Emadi, and J. Stake, "An electro-thermal HBV model," in IEEE MTT-S Int. Microw. Symp. Dig., Jun. 2005, pp. 1151-1153.

[41] J. R. Jones, G. B. Tait, S. H. Jones, and D. S. Katzer, "DC and large-signal time-dependent electron transport in heterostructure devices: An investigation of the heterostructure barrier varactor," IEEE Trans. Electron Devices, vol. 42, no. 6, pp. 1070-1080, Jun. 1995.

[42] D. S. Ong and H. L. Hartnagel, "Enhanced $\mathrm{THz}$ frequency multiplier efficiency by quasi-ballistic electron reflection in doubleheterojunction structures," Europhys. Lett., vol. 81, no. 8, p. 48004, 2008.

[43] M. Ingvarson, B. Alderman, A. Olsen, J. Vukusic, and J. Stake, "Thermal constraints for heterostructure barrier varactors," IEEE Electron Device Lett., vol. 25, no. 11, pp. 713-715, Nov. 2004.

[44] A. Malko, A.-Y. Tang, J. Vukusic, T. Bryllert, H. Zhao, and J. Stake, "Thermal analysis of III-V HBV diode structures on InP, GaAs, silicon and diamond substrates," presented at the 38th Int. Conf. Infr. Millim. THz Waves, Sep. 2013, pp. 1-2.

[45] J. Liljedahl, T. Bryllert, and J. Vukusic, "Development of a HBV tripler for 0.6 THz," presented at the 21st Int. Symp. Space THz Technol., 2010.

[46] M. Saglam, K. Mutamba, A. Megej, C. Sydlo, H. L. Hartnagel, and I. Daumiller, "Influence of polarization charges in $\mathrm{Al}_{0.4} \mathrm{Ga}_{0.6} \mathrm{~N} / \mathrm{GaN}$ barrier varactors," Appl. Phys. Lett., vol. 82, no. 2, pp. 227-229, Jan. 2003.

[47] N. Tanuma, S. Yokokura, T. Matsui, and M. Tacano, "Capacitance analysis of $\mathrm{Al}_{0.25} \mathrm{Ga}_{0.75} \mathrm{~N} / \mathrm{GaN}$ heterostructure barrier varactor diodes," Phys. Status Solidi $C$, vol. 2 , no. 7, pp. 2692-2695, May 2005.

[48] J. G. Champlain, R. Magno, M. Ancona, H. Newman, and J. Bradboos, "InAs-based heterostructure barrier varactor diodes with $\mathrm{In}_{0.3} \mathrm{Al}_{0.7} \mathrm{As}_{0.4} \mathrm{Sb}_{0.6}$ as the barrier material," Solid-State Electron., vol. 52, no. 11, pp. 1829-1832, Oct. 2008.

[49] S. Arscott, P. Mounaix, and D. Lippens, "Substrate transfer process for InP-based heterostructure barrier varactor devices," J. Vac. Sci. Technol. B, vol. 18, no. 1, pp. 150-155, Jan. 2000.

[50] N. Alijabbari, M. F. Bauwens, and R. M. Weikle, "Design and characterization of integrated submillimeter-wave quasi-vertical Schottky diodes," IEEE Trans. THz Sci. Technol., vol. 5, no. 1, pp. 73-80, Jan. 2015. 
[51] M. H. T. Dastjerdi, A. Sanz-Velasco, J. Vukusic, E. L. Kollberg, M. Sadeghi, and J. Stake, "InGaAs/InAlAs/AlAs heterostructure barrier varactors on silicon substrate," IEEE Electron Device Lett., vol. 32, no. 2, pp. 140-142, Feb. 2011.

[52] A. Malko, T. Bryllert, J. Vukusic, and J. Stake, "Silicon integrated InGaAs/InAlAs/ AlAs HBV frequency tripler," IEEE Electron Device Lett., vol. 34, no. 7, pp. 843-845, May 2013.

[53] A. Y. Tang, V. Drakinskiy, K. Yhland, J. Stenarson, T. Bryllert, and J. Stake, "Analytical extraction of a Schottky diode model from broadband S-parameters," IEEE Trans. Microw. Theory Techn., vol. 61, no. 5, pp. 1870-1878, May 2013.

[54] J. Stake, "Planar heterostructure barrier varactor diodes for millimetre wave applications," Ph.D. dissertation, Chalmers Univ. Technol., Gothenburg, Sweden, 1999.

[55] J. Vukusic, T. Bryllert, T. A. Emadi, M. Sadeghi, and J. Stake, "A 0.2-W heterostructure barrier varactor frequency tripler at $113 \mathrm{GHz}$," IEEE Electron Device Lett., vol. 28, no. 5, pp. 340-342, May 2007.

[56] Q. Xiao, J. L. Hesler, Y. Duan, T. W. Crowe, and R. M. Weikle, II, "A 300-GHz heterostructure barrier varactor (HBV) frequency septupler," in Proc. IRMMW-THz, Sep. 2005, pp. 80-81.

[57] N. Alijabbari, M. F. Bauwens, and R. M. Weikle, "160 GHz balanced frequency quadruplers based on quasi-vertical Schottky varactors integrated on micromachined silicon," IEEE Trans. THz Sci. Technol., vol. 4, no. 6, pp. 678-685, Nov. 2014.

[58] A. Maestrini et al., "A frequency-multiplied source with more than $1 \mathrm{~mW}$ of power across the 840-900-GHz band," IEEE Trans. Microw. Theory Techn., vol. 58, no. 7, pp. 1925-1932, Jul. 2010.

[59] T. W. Crowe, J. L. Hesler, S. A. Retzloff, and D. S. Kurtz, "Higher power multipliers for terahertz sources," presented at the 41st Int. Conf. Infr. Millim., Terahertz waves, 2016, p. 1.

[60] D. W. Porterfield, "High-efficiency terahertz frequency triplers," in IEEE MTT-S Int. Microw. Symp. Dig., Mar. 2007, pp. 337-340.
[61] E. Schlecht, "200, 400 and $800 \mathrm{GHz}$ Schottky diode 'substrateless' multipliers: Design and results," in IEEE MTT-S Int. Microw. Symp. Dig., vol. 3. May 2001, pp. 1649-1652.

[62] T. Bryllert, A. Malko, J. Vukusic, and J. Stake, "A 175 GHz HBV frequency quintupler with $60 \mathrm{~mW}$ output power," IEEE Microw. Wireless Compon. Lett., vol. 22, no. 2, pp. 76-78, Feb. 2012.

[63] J. V. Siles et al., "A high-power 105-120 GHz broadband on-chip power-combined frequency tripler," IEEE Microw. Wireless Compon. Lett., vol. 25, no. 3, pp. 157-159, Feb. 2015.

[64] R. Dahlbäck, V. Drakinskiy, J. Vukusic, and J. Stake, "A compact 128-element Schottky diode grid frequency doubler generating 0.25 W of output power at $183 \mathrm{GHz}$," IEEE Microw. Wireless Compon. Lett., vol. 27, no. 2, 2017. DOI: 10.1109/LMWC.2017.2652857

[65] J. B. Hacker, A. L. Sailer, B. Brar, G. Nagy, R. L. J. Person, and J. A. Higgins, "A high-power W-band quasi-optical frequency tripler," in IEEE MTT-S Int. Microw. Symp. Dig., vol. 3. Jun. 2003, pp. 1859-1862.

[66] J. Stake, T. Bryllert, J. Vukusic, and A. Olsen, "Development of high power HBV multipliers for millimeter wave applications," Proc. SPIE, vol. 6739, p. 67390 U, Oct. 2007.

[67] M. Saglam et al., "High-performance 450$\mathrm{GHz}$ GaAs-based heterostructure barrier varactor tripler," IEEE Electron Device Lett., vol. 24, no. 3, pp. 138-140, Mar. 2003.

[68] A. Malko, T. Bryllert, J. Vukusic, and J. Stake, "High efficiency and broad-band operation of monolithically integrated W-Band HBV frequency tripler," presented at the 24th Int. Conf. Indium Phosph. Rel. Mater., Aug. 2012, pp. 92-94.

[69] P. F. X. Neumaier et al., "Molecular spectroscopy with a compact $557-\mathrm{GHz}$ heterodyne receiver," IEEE Trans. THz Sci. Technol., vol. 4, no. 4, pp. 469-478, Jul. 2014.

[70] A. V. Raisanen et al., "A single barrier varactor quintupler at $170 \mathrm{GHz}$," IEEE Trans. Microw. Theory Techn., vol. 43, no. 3, pp. 685-688, Mar. 1995.
[71] B. Diamond, "Idler circuits in varactor frequency multipliers," IEEE Trans. Circuit Theory, vol. 10, no. 1, pp. 35-44, Mar. 1963.

[72] T. Bryllert et al., "11\% efficiency $100 \mathrm{GHz}$ InP-based heterostructure barrier varactor quintupler," Electron. Lett., vol. 41, no. 3, pp. 131-132, Feb. 2005.

[73] T. David et al., "Monolithic integrated circuits incorporating InP-based heterostructure barrier varactors," IEEE Microw. Wireless Compon. Lett., vol. 12, no. 8, pp. 281-283, Aug. 2002.

[74] Q. Xiao, J. L. Hesler, T. W. Crowe, R. M. Weikle II, Y. Duan, and B. Deaver, "Highefficiency heterostructure-barrier-varactor frequency triplers using AlN substrates," in IEEE MTT-S Int. Microw. Symp. Dig., May 2005, pp. 443-446.

[75] S. Hollung, J. Stake, L. Dillner, and E. Kollberg, "A 141-GHz integrated quasioptical slot antenna tripler," in Proc. IEEE Antennas Propag. Soc. Int. Symp., vol. 4. Jun. 1999, pp. 2394-2397.

[76] C. F. Jou, W. W. Lam, H. Z. Chen, K. S. Stolt, N. C. Luhmann, and D. B. Rutledge, "Millimeter-wave diodegrid frequency doubler," IEEE Trans. Microw. Theory Techn., vol. 36, no. 11, pp. 1507-1514, Nov. 1988.

[77] G. Hrubó, R. Dahlbäck, and J. Stake, "Comparison of full 3D and unit cell models for waveguide-embedded frequency multiplier arrays," IEEE Microw. Wireless Compon. Lett., vol. 26, no. 11, pp. 954-956, Nov. 2016

[78] M. Li, K. Krishnamurthi, and R. G. Harrison, "A fully distributed heterostructure-barrier varactor nonlinear transmission-line frequency multiplier and pulse sharpener," IEEE Trans. Microw. Theory Techn., vol. 46, no. 12, pp. 2295-2301, Dec. 1998.

[79] H. Xu, Y. Duan, J. L. Hesler, T. W. Crowe, and R. W. Weikle, "Subharmonically pumped millimeter-wave upconverters based on heterostructure barrier varactors," IEEE Trans. Microw. Theory Techn., vol. 54, no. 10, pp. 3648-3653, Oct. 2006.

\section{ABOUT THE AUTHORS}

Jan Stake (Senior Member, IEEE) was born in Uddevalla, Sweden, in 1971. He received the M.Sc. degree in electrical engineering and the Ph.D. degree in microwave electronics from Chalmers University of Technology, Göteborg, Sweden, in 1994 and 1999 , respectively.

In 1997, he was a Research Assistant with the University of Virginia, Charlottesville, VA, USA. From 1999 to 2001, he was a Research Fellow with the Millimetre Wave Group at the Rutherford Appleton Laboratory, Didcot, U.K.

He then joined Saab Combitech Systems $A B$ as a Senior RF/Microwave Engineer, where he remained until 2003. From 2000 to 2006, he held various academic positions with Chalmers University of Technology, and from 2003 to 2006, he was also the Head of the Nanofabrication Laboratory, Department of Microtechnology and Nanoscience (MC2). During 2007, he was a Visiting Professor with the Submillimeter Wave Advanced Technology (SWAT) Group at Caltech/JPL, Pasadena, CA, USA. He is currently Professor and Head

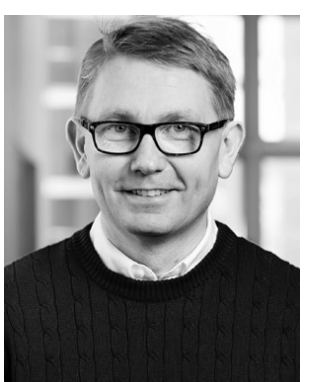

of the Terahertz and Millimetre Wave Laboratory at Chalmers University of Technology. He is also cofounder of Wasa Millimeter Wave AB, Göteborg, Sweden. His research involves graphene electronics, high-frequency semiconductor devices, THz electronics, submillimeter wave measurement techniques ("THz metrology"), and THz applications in biology and medicine.

Prof. Stake serves as Editor-in-Chief for the IEEE Transactions on Terahertz Science and Technology.

\begin{abstract}
Aleksandra Malko was born in 'Swidnica, Poland, in 1984. She received the M.Sc. degree in engineering from the Department of Electronics, Microsystems and Photonics, Wrocław University of Technology, Wrocław, Poland, in 2008 and the Ph.D. degree in microtechnology and nanoscience from Chalmers University of Technology, Göteborg, Sweden, in 2015. Her Ph.D. dissertation was on silicon integrated HBVbased frequency multipliers.
\end{abstract}

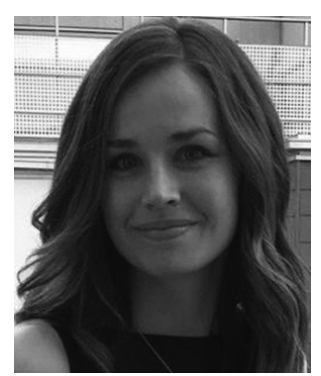


During her four months as a Postdoctoral Researcher at MC2, she was involved in the development of metamorphic Schottky diodes for high-frequency mixers and multipliers. Currently, she is with United Monolithic Semiconductors GmbH, Ulm, Germany, where she works on the development of GaAs and GaN HEMTs. Her research interests include $\mathrm{III}-\mathrm{Vs}, \mathrm{III}-\mathrm{N}$ and silicon process technologies, methods of integrating compound semiconductors on silicon, and characterization methods for power devices.

Tomas Bryllert (Member, IEEE) was born in Vaxjo, Sweden, in 1974. He received the M.S. degree in physics and the Ph.D. degree in semiconductor physics from Lund University, Lund, Sweden, in 2000 and 2005, respectively.

In 2006, he joined the Microwave Electronics Laboratory at Chalmers University of Technology, Göteborg, Sweden, where his main research interest was device and circuit technology for $\mathrm{THz}$ frequency multipliers. From 2007 to 2009 , he was with the

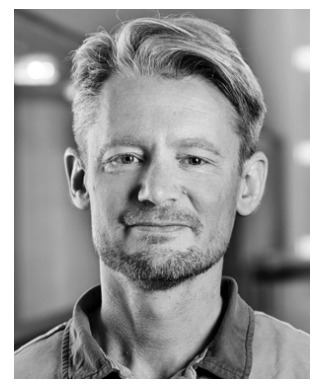

Submillimeter Wave Advanced Technology (SWAT) group at the Jet Propulsion Laboratory, California Institute of Technology, Pasadena, CA, USA, working on THz imaging and radar systems. He is currently with the Terahertz and Millimetre Wave Laboratory in the Department of Microtechnology and Nanoscience (MC2), Chalmers University of Technology. He is also cofounder and Chief Executive Officer (CEO) of Wasa Millimeter Wave AB, a company that develops and fabricates millimeter-wave products.

Josip Vukusic received the Diploma and Ph.D. degrees in photonics from Chalmers University of Technology, Göteborg, Sweden, in 1997 and 2003, respectively.

He has been with the Terahertz and Millimetre Wave Laboratory, Department of Microtechnology and Nanoscience, Chalmers University of Technology, since 2004, where he is involved in the modeling, fabrication and characterization of $\mathrm{THz}$ technology for various applications. He is also one of the founders of Wasa Millimeter Wave $A B$, which markets high-frequency products.

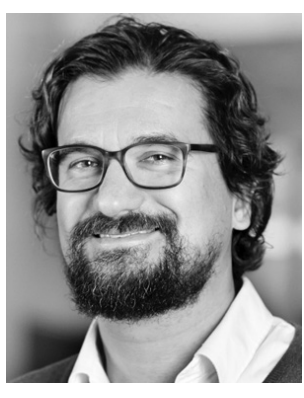

